\section{Hauptsache üben - Technik egal!}

\section{Zur Asthmaschulung gehört auch der Hinweis auf eine Optimierung der Atemtechnik - im Alltag wie im Bedarfsfall. Der Vergleich zweier Techniken zeigt, dass auch eher unspezifische Übungen hilfreich sind.}

$M$ ehrere Studien konnten bereits zeigen, dass gezielte Atemtechniken den Bedarf an kurz wirksamen $\beta_{2}$ Sympathomimetika reduzieren und die Lebensqualität von Asthmakranken verbessern können. Australische Forscher untersuchten jetzt im Rahmen einer randomisierten doppelblinden Studie über 30 Wochen den Effekt zwei verschiedener Übungsprogramme auf die Asthmasymptomatik. Nach einer zweiwöchigen Einführungsphase wurden die 57 Patienten in zwei Gruppen aufgeteilt. Über Video erhielten sie Anleitungen für Übungen - entweder für ein spezielles Übungsprogramm, das die flache Nasenatmung fördern sollte (Gruppe A), oder für ein eher unspezifisches Bewegungstraining des Oberkörpers (Gruppe B). In beiden Fällen wurden die Teilnehmer angehalten, die Übungen zweimal pro Tag durchzuführen. Nach 16 Wochen wurde versucht, die Glukokortikoiddosis in zwei Stufen zu reduzieren.

Zwischen den beiden Gruppen bestanden weder nach zwölf noch nach 28 Wochen klinisch relevante Unterschiede. Der Lebensqualitätsscore blieb in beiden

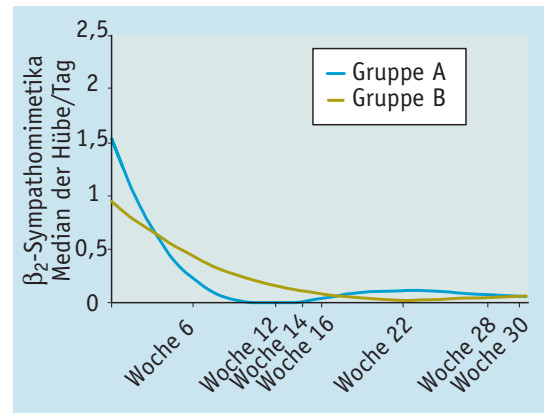

Sowohl spezielle Atemtechniken (Gruppe A) als auch unspezifische Oberkörperübungen (Gruppe B) reduzieren den Bedarf an $\beta_{2}$-Sympathomimetika.

\title{
Asthmatherapie kein Risiko für das Baby
}

\section{Asthma der Mutter kann zu Frühgeburtlichkeit, intrauteriner Wachs- tumsretardierung und Präeklampsie führen. Bei guter Einstellung ist das Risiko dieser Komplikationen allerdings nicht erhöht. Dagegen ab- zuwägen ist ein mögliches teratogenes Risiko der Therapeutika.}

nhalative Glukokortikoide sind ein Eckpfeiler der Asthmatherapie. Aus Tierversuchen gibt es Hinweise auf teratogene Effekte dieser Substanzgruppe. Diese konnten jedoch in drei populationsbasierten Beobachtungsstudien mit insgesamt 2.534 Frauen bisher nicht bestätigt werden. Es war unter Budesonid nicht öfter zu Fehlbildungen oder anderen Komplikationen gekommen.

Britische Ärzte wollten es nochmals genau wissen und überprüften im Rahmen der prospektiven randomisierten Studie START (,inhaled steroid treatment as regular therapy") die Auswir- kungen einer Glukokortikoidtherapie bei 7.241 Frauen mit mildem bis mäßigem Asthma. In den drei Jahren der ersten Studienphase erhielten die Patientinnen zusätzlich zu ihrer üblichen Therapie entweder $400 \mu \mathrm{g}$ Budesonid oder Plazebo über einen Inhalator. In einer anschließenden zweiten Phase wurden alle Patienten über zwei Jahre mit dem Glukokortikoid behandelt. Während der ersten Phase traten 219 Schwangerschaften auf, davon 102 in der Budesonidgruppe und 117 unter Plazebo, in der zweiten Phase 94. Der Anteil gesund geborener Kinder betrug in der Budesonidgruppe $81 \%$ und
Gruppen unverändert, ebenso Lungenfunktionsparameter und bronchiale $\mathrm{Hy}$ perreagibilität. In beiden Trainingsgruppen nahm aber der Verbrauch an Bedarfsmedikation drastisch um $86 \%$ ab und auch die Dosis an inhalativen Glukokortikoiden konnte um die Hälfte reduziert werden - wiederum ohne signifikanten Unterschied zwischen den beiden Gruppen. Die Autoren favorisieren Atemübungen besonders für Asthmapatienten, die häufig zu Bedarfsmedikamenten greifen, da in der Reduktion kurz wirksamer $\beta_{2}$-Sympathomimetika der größte Nutzen des Trainings zu liegen scheint.

Fazit: Durch Atemübungen können Asthmakranke ihren Bedarf an $\beta_{2}$-Sympathomimetika und Glukokortikoiden reduzieren, ohne dafür mit einer schlechteren Lungenfunktion zu bezahlen. Die Übungen sollten regelmäßig erfolgen, spezielle Techniken scheinen nicht unbedingt notwendig zu sein.

Slader CA et al. Double blind randomised controlled trial of two different breathing techniques in the management of asthma. Thorax 2006; 61: 651-6 war damit dem Anteil von 77\% in der Plazebogruppe vergleichbar. Auch bei der Komplikationsrate ergab sich kein signifikanter Unterschied, sie betrug 19\% unter Glukokortikoidgabe und 23\% unter Plazebo. Spontanaborte traten unter Verum bei $12 \%$ der Fälle auf, unter Plazebo bei $9 \%$. In der Budesonidgruppe wurden sieben Frühgeburten und 28 Kaiserschnitte verzeichnet, während es in der Plazebogruppe zu acht Frühgeburten und vier Kaiserschnitten kam.

Fazit: Diese prospektive Studie bestätigt, dass sich eine langjährige Asthmatherapie mit einem niedrig dosierten inhalativen Glukokortikoid offenbar nicht negativ auf eine Schwangerschaft auswirkt. iw

Silverman $\mathbf{M}$ et al. Outcome of pregnancy in a randomized controlled study of patients with asthma exposed to budesonide. Ann Allergy Asthma Immunol 2005; 95: 566-70 